Aus der König1. Universitäts-Frauenklinik zu Halle a. S.

Direktor: Geh. Med.-Rat Prof. Dr. Veit.

\title{
Die Herkunft der Blutung bei der Ruptur der Tubenschwangerschaft.
}

\author{
Von \\ Dr. Y. Taniguchi, Kumamoto-Japan.
}

(Hierzu Tafel $\nabla$ und 2 Textfignren.)

Auf die Anregung von Herrn Geheimrat Prof. Dr. J. Veit habe ich mich mit der Frage der Herkunft des Blutes bei Ruptur der Tubenschwangerschaft beschäftigt und glaube meiner Aufgabe gerecht zu werden, wenn jch folgende 3 Fragen beantworte:

1. Woher stammt die Blutung, aus der die Graviditas tubaria sich beinahe verblutet hat? Aus den Arterien oder aus den Venen oder aus intervillösen Räumen?

2. Tritt die Blutung vor der Ruptur der schwangeren Tube oder als Folge derselben auf?

3. Was ist die Herkunft der Blutung bei der Ruptur der schwangeren Tube?

Wenn eine Tubenschwangerschaft zum Bersten kommt, so findet oft eine sehr starke Blutung statt, die das ganze Abdomen mit flüssigem und koaguliertem Blute in kolossalen Mengen überschwemmt, so dass manche Frauen an Verblutung sterben, falls nicht rechtzeitig operative Hilfe eintritt oder das Blut gewöhnlich hinter dem Uterus sich abkapselt, was man als Haematocele retrouterina bezeichnet. Erfolgt die Blutung in das Ei in der Art, dass sie sich auf die Höhle des Fruchtsackes beschränkt, so nennen wir dies eine Haematosalpinx e graviditate, Tubenmole oder Haematoma tubae. Das abgestorbene Ei kann dann ausgestossen werden, es kommt zum Tubenabortus. Das Blut setzt sich an den Tuben, besonders an dem ampullären Tubenende zwiebelschalenartig an und bildet hier einen Tumor. Dieses nennt man dann eine peri- 
tubare Hämatocele ${ }^{1}$ ). Zerreisst der tubare Fruchtsack nicht an seiner freien Oberfläche, sondern an dem gegen das Jjigamentum latum zu gelegenen und nicht von der Serosa bekleideten Wandabschnitt, so bahnt sich das Blut einen Weg in die beiden Blätter und bildet hier das Haematoma parauterinum oder das subseröse Hämatom [Kuhn²)].

Bevor ich an die Frage nach der Entstehung der Blutungen herangehe, erscheint es mir nicht überflüssig, kurz die Theorie und Erklärung der Ruptur bei der Tubargravidität zu behandeln.

Unter einem "chronischen" Verlauf der Ruptur verstehe ich die eigentümliche Erscheinung, dass man bei der Laparotomie eine Zerreissung der schwangeren Tube findet, ohne dass lebensbedrohliche Erscheinungen vorausgegangen wären. Auch mit dem Ausdruck "Usur" meint man, wenn ich von den Fällen der sogenannten sekundären Bauchschwangerschaft absehe, etwa das gleiche. Man spricht von Usur, sowohl bei diesem langsamen Verlauf der klinischen Erscheinungen als auch dann, wenn die Eröffnung der Tube ausserhalb der Plazentarstelle erfolgt und eine Störung in der weiteren Entwicklung des Eies ausbleibt, wenn es also zur sekundären Bauchschwangerschaft kommt. Man findet bei allmählicher Verdünnung der Wand in der schwangeren Tube eine kleine Oeffnung, ohne dass vor der Operation irgend etwas auf diese Störung hinwies. Die gleiche anatomische Bedeutung hat das Vorkommen mehrfacher Rupturen der Tube und das Bild, welches Aschoff3) als versteckte Ruptur bezeichnet. Die Erklärung hierfür ist mit dem Platzen der Tube durch den Druck des wachsenden Eies und mit dem Durchpressen bzw. dem Arrodieren nur dann vereinbar, wenn man annimmt, dass die Lücken sofort nach der Eröffnung durch eindrängende Zotten verschlossen werden, so dass keinerlei stürmische Erscheinungen dabei aufzutreten brauchen. Will man den Ueberdruck im intervillösen Raum, sei es durch Torsion oder durch Zottenverschleppung annehmen, so kann man diese Erscheinungen nur dadurch deuten, dass, während ein Teil des intervillösen Raumes den Störungen aus-

1) A. Martin, Pathologie und Therapie der Frauenkranlheiten. 4. Aufl. 1907. S. 403 .

2) Kuhn, Ueber Blutergüsse in die beiden Mutterbänder. Inaug.-Diss. Zürich 1874.

3) Aschoff, Dieses Arohiv. 1900. Bd. 60. S. 523. - Zentralbl. f. allgem. Pathol. u. pathol. Anat. 1901. Nr. 11 u. 12. S. 449. 
gesetzt ist, die Nachbarzotten nicht leiden. Aus ihnen kann das Blut noch in die Vene abfliessen.

Die Herkunft des Blutes bei diesen chronischen Rupturen, bei der Usur und bei der multiplen Ruptur, ist von besonderem Interesse, weil die Blutung nicht lebensbedrohlich stark wird. Bei jeder Erklärung der Zerreissung der Tubenwand muss man mit den verschiedensten Möglichkeiten der Herkunft des Blutes rechnen. Es kann aus einer Arterie der Tubenwand stammen, welche zerriss, oder eventuell auch aus der Vene oder endlich aus dem intervillösen Raume. Bei all' diesen Erklärungen aber muss man auch die Erklärung dafür geben können, dass die Blutung nach einer gewissen Zeit oder auch schnell ausnahmsweise sich begrenzen kann. Nicht jede Blutung führt zum Tode. Sofortiger Stillstand der Blutung ist am leichtesten erklärlich bei Herkunft des Blutes aus dem intervillösen Raum.

Auch ist es von Wichtigkeit bei der Frage der Entstehung: der Ruptur an die Fälle zu denken, bei denen die Entwicklung des Eies überhaupt nicht gestört wurde, sondern bei denen nach der Eröffnung der Tube das Ei sich ungestört weiter entwickelt als sekundäre Bauchschwangerschaft. In solchen Fällen sitzt die Usur niemals an der Plazentarstelle, sondern dort, wo das Chorion laeve sich entwickelt hat. Ich erkläre mir die Ruptur in diesen Fällen durch vorübergehende Tubenzusammenziehungen und damit eintretende abnorme Spannung in dem Ei und in der Tube. Wenn dann die ödematösen Muskelfasern der Tube schon zum Teil auseinandergewichen sind, wird es bei Zusammenziehungen leicht zur Zerreissung der Wand kommen können. Bei diesen Arten der Zerreissung der Tubenwand kommt es so gut wie niemals zu Blutungen in die Bauchhöhle.

Bei der plötzlichen Ruptur haben wir es mit einem explosionsartigen breiten Aufreissen der äusseren Fruchtkapselwand zu tun, und es bricht nicht nur die Tubenwand auf, sondern auch die Reflexa und die Eihäute zerreissen. Während bei der Usur die Blutung in den meisten Fällen fehlt, ist sie bei der Ruptur in der Regel sehr häufig und stellt immer einen äusserst stürmischen Vorgang dar. In vereinzelten Fällen wurde die Blutung so stark, dass in kurzer Zeit der Tod erfolgte. So konnte Fehling ${ }^{1}$ ) in 1 Falle den Tod $3 / 4$ Stunden naeh den ersten Symptomen beobachten.

1) Fehling, Zeitsehr. f. Geb. u. Gyn. 1898. Bd. 38. S. 67. 
240 Taniguchi, Ueber Blutung bei der Ruptur der Tubenschwangerschaft.

Als Ursache des äusseren Fruchtkapselaufbruches nahm man früher allgemein ${ }^{1}$ ) an, dass die Fruchtkapselwand durch den rein mechanischen Druck des wachsenden Eies verdünnt wurde und dadurch eine Ruptur hervorgerufen werden musste. Neuerdings muss man dies als zweifelhaft bezeichnen, da man eine regelmässige Weichheit der schwangeren Tube feststellen kann und festgestellt hat. Nur interkurrente Zusammenziehungen der Muskelwand der Tube kann hieran etwas ändern, nur dadurch entsteht ein abnormer Druck im Ei. Immerhin finden noch viele Autoren den Grund in anderen Ursachen. Werth ${ }^{2}$ ) änssert sich dazu, indem er sagt, dass der äussere Kapselaufbruch neben der passiven Dehnung durch das wachsende $\mathrm{Ei}$ auch durch Arrosionserscheinungen, die in dem mütterlichen Gewebe überall an dessen Berührungsfläche mit dem fötalen Fktoderm auftreten, beruhen. Aber meines Erachtens kommt auch die Staung in der Tube und dadurch bedingte Blutung in die Gewebe als erklärendes Moment in Betracht. A. Martin ${ }^{3}$ ) wies als Ursache für eine plötzliche Blutstauung die Torsion nach. Weiter verglich man die Ursache der Ruptur mit der Erklärung für den Blutgehalt des intervillösen Raumes.

Kühne $e^{4}$ ) ist der Ansicht, dass die Eröffnung der Gefässe durch die fötalen Zellen ein pathologischer Zustand sei. Kreis $\mathrm{ch}^{5}$ ), Ulesko-Stroganowa ${ }^{6}$ ), Füth ${ }^{7}$ ), Petersen ${ }^{8}$, , Voigt ${ }^{9}$ ) u. a. hatten ein Durchwachsen von ganzen Zotten aus dem intervillösen Raume

1) S. z. B. Kussmaul, Von dem Mangel, der Verkümmerung und̉ Verdoppelung der Gebärmutter, von der Nachempfängnis und der Ueberwanderung des Eies. S. 337. Würzburg 1859.

2) Werth, Winckel's Handbuch. 2. Aufl. Bd. 2. T. 2. S. 690.

3) A. Martin u. Orthmann, A. Martin's Krankheiten des Eiters. S. 301. Leipzig 1895.

4) Kühne, Beiträge zor Anatomie der Tubenschwangerschaft. Habilit. Marburg 1899.

5) Kreisch, Monatsschr. f. Geburtsh. u. Gyn. 1899. Bd. 9. S. 794.

6) Ulesko-Stroganowa, Monatsschr. f. Geburtsh. u. Gynäk. 1900. Bd. 11. S. 104. Bd. 12. S. 710.

7) Füth, Monatsschr. f. Geburtsh. u. Gyn. 1898. Bd. 8. S. 390 und dieses Arch. 1901. Bd. 63. S. 97.

8) Petersen, Beiträge zur pathologischen Anatomie der graviden Tube. Berlin 1902. S. Karger.

9) Voigt, Dieses Arch. 1903. Bd. 68. S. 642. 
durch das Bindegewebe in die Gefässe angenommen und infolge der Zerreissung der Gefässe eine Blutung festgestellt. Obige Autoren hatten diese Vorgänge durch die aggressive Tätigkeit der Zotten erklärt. Leopold ${ }^{1}$ ) schrieb den aktiven Vorgang nur der speziellen Tätigkeit der Synzytiumzellen, Heinsius ${ }^{2}$ ) der der Langhanszellen, Aschoff (l. c.) der der Chorionepithelzellen zu. Diese Fähigkeit betrachtet man als eine histologisch auf das mütterliche Gewebe einwirkende, gerade wie Osteoklasten auf Knochen [Stöhr $\left.{ }^{3}\right)$ ]. Heinsius (l. c.) nimmt ein Durchbrechen der Zotten in die Venen an, konnte aber daneben ein Einbrechen der Zotten in die Arterien nicht sehen. Kiwisch ${ }^{4}$, Virchow ${ }^{5}$, Waldeyer ${ }^{6}$ ), Pfannenstiel $^{7}$ ), Kroemer ${ }^{8}$ ) u. a. stellen die aggressive Tätigkeit seitens der fötalen Zellen in Abrede, indem sie sagen, dass diese in den Venen nicht die Gefässwand anfressen, sondern unmittelbar vom mütterlichen Blute bespült werden. Couvelaire ${ }^{9}$ ) sieht auch diese Erscheinung weniger in der zerstörenden Wirkung der Zotten als in der schwachen Anlage der Dezidua.

V eit ${ }^{10}$ ) hält die angebliche Zerstörung der Gefässwand seitens der fötalen Zellen für nicht erwiesen. Er erklärt das weite Tiefeindringen der Zotten und des Zottenepithels in die Tubenwand sowie in die Vene für eine Folge der von ihm so bezeichneten sogen. "Deportation der Chorionzotten". Diese Tatsache ist vor ihm und nach ihm von den verschiedensten Autoren, wie Leopold ${ }^{11}$ ),

1) Leopold, Dieses Arch. Bd. 59. S. 516.

2) Heinsius, Zeitschr. f. Geburtsh. u. Gyn. 1901. Bd. 46. S. 385 und Zentralbl. f. Gyn. 1903. S. 855.

3) Stöhr, Lehrbuch der Histologie. 1912. Aufl. 15. S. 173. S. 91 .

4) Kiwisch, s. Waldeyer, Ueber den Plazentarkreislauf des Menschen.

5) Virchow, Gesam. Abhandlungen zur wissenschaftlichen Medizin. Frankfurt 1856.

6) Wald eyer, Sitzungsber. d. Königl. Preuss. Akademie d. Wissensoh. zu Berlin 1887.

7) Pfannenstiel, Winckel's Handbuch. Bd. 1. H. 1. S. 247.

8) Kroemer, Dieses Arch. 1903. Bd. 68. S. 57.

9) Couvelaire, s. Veit, Verhandl. d. deutschen Gesellsch. f. Gyn. X. Kongr. 1903. S. 24.

10) Veit, Zeitschr. f. Geburtsh. u. Gyn. 1901. Bd. 44. S. 465. - Verhandlungen d, deutschen Gesellsch. f. Gyn, X. Kongr. 1903. S. 20. - Die Verschleppung der Chorionzotten. Wiesbaden 1905.

11) Leopold, Winckel's Handbuch. Bd. 2. T. 2. S. 677. 
242 Taniguchi, Ueber Blutung bei der Ruptur der Tubenschwangerschaft.

Pfannenstiel (l. c.), Schmorlin), Hitschmann $\left.{ }^{2}\right)$, Poten $^{3}$ ), Fellner ${ }^{4}$, Fromme ${ }^{5}$ ), Kiutsi ${ }^{6}$ ) a. a. beobachtet worden. Die oben erwähnten Theorien über die Ursache der Ruptur lehnt er ab, er erkennt weder den Wachstumsdruck ohne Kontraktion noch die Zerstörungskraft des Eies an: Wachstumsdruck ersetzt er durch abnormen Druck im Ei, und eine der Ursachen hierfür sieht er in dem häufigen Auftreten von Kontraktionen; eine weitere in den von Kiatsi erwiesenen Zeichen.

Aschoff (1. c.) gibt als Grund für diese Blutungen stärkere Schwankungen des Blutdrucks oder Kontraktionen des noch erhaltenen Teiles des Muskelringes an, weil die Widerstandskraft der Wandung der tubaren Gefässe geringer ist als die der uterinen, und die Zerreissung der in der Plazentarbildung einbezogenen grösseren Gefässe für die schwangere Tube eine plötzliche Blutdrucksteigerung zur Folge hat.

Kiutsi (l. c.) hat durch ausgezeichnete genaue Untersuchungen die Veit'sche Ansicht, dass die Ursache der Ruptur weder durch den Druck des wachsenden Eies noch durch die zerstörende Kraft des Wachstums der Zotten bedingt ist, gestützt und festgestellt, dass sie durch den Blutüberdruck infolge von Verstopfung der abführenden Venen verursacht wird.

Ferner kann man es nicht leugnen, dass für einzelne Fälle die Torsion der schwangeren Tube die Ursache der Blutung ist. Weiterhin kommt die äussere, mehr oder weniger accidentelle Einwirkung - Stoss, Fall, grössere und starke Anstrengung der Bauchpresse, Druck auf den Leib - in Betracht. Aber alles dies darf uns nur als Nebenursache gelten, weil, wenn auch diese Ursachen fehlen, die Ruptur doch eintreten kann.

Es ist nun von Interesse zu fragen, woher das Blut bei der Blutung in die Bauchhöhle stammt. Wenn der Druck des wachsenden Eies und die zerstörende Kraft der Zotten oder der arterielle Ueberdruck des intervillösen Raumes zur Zerreissung führt, kann die Blutung aus einer zerrissenen Arterie, aus einer zerrissenen

1) Schmorl, Pathol.-anat. Untersuchungen über puerperale Eklampsie. Leipzig 1893.

2) Hitschmaun, Zeitschr. f. Geburtsh. u. Gyn. Bd. 53. S. 1.

3) Poten, Zeitschr. f. Geburtsh. u. Gyn. Bd. 66: S. 590.

4) Fellner, Dieses Arch. Bd. 74. S. 481.

5) Fromme, Zentralbl. f. Gyn. 1910, S. 347.

6) Kiutsi, Dieses Arch. 1912. Bd. 95. S. 493. 
Vene oder aus dem intervillösen Raume stammen. Fehling ${ }^{1}$ ) hat gesagt, dass aus einer solchen kleinen, oft nur 1-2 $\mathrm{mm}$ grossen Oeffnung der Tube eine tödliche Verblutung erfolgen kann, während bei den ungleich viel grösseren Zerreissungen des puerperalen Uterus selten eine tödliche Verblutung erfolgt. Nach Werth ${ }^{2}$ ) ist die Blutung aus den peripher unter dem Eiboden gelegenen Arterien und den an sich nicht starken Tubenästen deshalb so stark und anhaltend, weil wegen des kurzen Verlaufs von ihrem Ursprunge aus dem utero-ovariellen Gefässstamme in ihnen ein starker Seitendruck herrscht. Kiutsi ${ }^{3}$ ) betont, dass die foudroyante Blutung bei der Tubenruptur aus den Arterien stammt, welche doppelte Herkunft von ihren Stammarterien und ungenügende Gesamtquerschnittsvermehrung im Eibett haben. Richtiger scheint die Ansicht Veit's4) zu sein, der die Blutung auf Staung infolge Verstopfung der aus den intervillösen Räumen abführenden Venen durch Zottẹn und Zottenteilchen zurückführt. Er erklärt folgendes: "Hier, bei der Tubenschwangerschaft, wird eine Vene dicht unter dem Peritoneum mit Zotten ganz erfüllt, zwischen der Verstopfungsstelle und dem intervillösen Raum staut sich Blut in den Venen, bis eine derselben und mit ihr das Peritoneum zerreisst, und nun ist an dieser Stelle der Widerstand viel geringer als an den übrigen Stellen des intervillösen Raumes; alles Blut strömt aus den Arterien fast direkt hierher, dazu kommt, dass vor der Zerreissung der Vene das Ei durch die Blutstaung in grösserer Ausdehnung sich abgelöst hat und es daher aus den abgelösten Stellen auch blutet. Damit wird der Blutabgang so stark, wie er bei einem uterinen Abort ist. Während aber bei diesem die Kontraktion der Wand die Blutung. schliesslich beherrscht, zieht in der gerissenen Tube die Kontraktion die Rissstelle auseinander, die Blutung dauert also nach der Zerreissung an. Diese Kombination von Umständen erklärt mir die foudroyante Stärke der Blutung bei mancher Ruptur der Tube."

Ich werde nun an der Hand eigener Beobachtungen zu untersuchen haben, woher die Blutung bei der Ruptur stammt, ob aus der Arterie oder der Vene oder aus dem intervillösen Raum.

1) Fehling, Verhandl. der deutschen Gesellsch. f. Gyn. 10. Kongr. 1903. S. 201.

2) Werth, Verhandl. der deutschen Gesellsch. f. Gyn. 10. Kongr. 1903. S. 99. - Winckel's Handbuch. Bd. 2. T. 2. S. 701.

3) Kiutsi, Dieses Arohiv. Bd. 95. S. 546.

4) Veit, Ueber Extrauteringravidität. Schlusswort. S. 213. 
244 Taniguchi, Ueber Blutung bei der Ruptur der Tubenschwangerschaft.

\section{Eigenes Material.}

Es stammt von einer rechtsseitig geborstenen Tubargravidität. Die Patientin wurde in der Veit'schen Klinik wegen der lebensgefährlichen Blutung sofort nach ihrer Einlieferung am 13. Juli 1913 operiert.

Frl. W. H., 25 Jahre alt, aus Halle. Primipara. Nr. 676. Aufgenommen am 13. VII., entlassen am 31. VII. 1913.

Anamnese: Eltern und 4 Geschwister gesund. Patientin wurde vor 2 Jahren in hiesige Klinik wegen Weissfluss aufgenommen, sonst war sie gesund. Erste Menstruation trat mit 16 Jahren auf, immer regelmässig, Dauer 5-6 Tage; letzte Menstruation am 11. VII. Am 12. VII. bekam Pat. in der Nacht heftige Schmerzen, Ohnmachtsanfälle und auch Fieber, daher wird sie am 13. VII. hier eingeliefert.

Jetzige Symptome: Heftige Schmerzen im Unterleib.

Aufnahmebefund: Stark anämische Patientin. Temperatur 38,1, Puls beschleunigt, klein, schwach. Brüste wenig entwickelt. Abdomen etwas aufgetrieben, starke Spannung und Empfindlichkeit auf Druck. Aus der Scheide geringe Blutung. Portio normal, ausserer Muttermund geschlossen. Uterus nach vorne, nicht vergrössert. An den Parametrien rechts und links Resistenzen, die nicht genau palpiert sind.

Diagnose: Extrauteringravidität.

Operation: Exstirpation einer rechtsseitigen Tubargraviditat und eines linksseitigen Ovarialtumors. Operateur: Privatdoz. Dr. Heynemann. Desinfektion des Abdomens in der üblichen Weise. Längssehnitt in der Mittellinie. In der Bauchhöhle reichlich Blut, das schon durch das Peritoneum hindurch schimmert. Die rechtsseitige Tubargravidität wird isoliert abgeklemmt, abgeschnitten und die Klemme durch Katgutunterbindungen ersetzt. Die Blutung steht nach Anlegung der Klemmen vollständig. Exstirpation der linksseitigen Ovarialcyste. Entfernung des Blutes aus der Bauchhöhle mit Tüchern. Schluss der Bauchwunde in der üblichen Weise in 3 Etagen. Während der Operation Kampfer und Kochsalz. Am 3. Tage nach der Operation Temperatursteigerung bis $38,3^{\circ}$. Die Wunde heilt per primam intentionem. Am 31. Vll. entlassen.

\section{Makroskopische Beschreibung der exstirpierten Adnexe.}

I. Die rechtsseitige schwangere Tube. Das Präparat, in Formalin gehärtet, ist $6,1 \mathrm{~cm}$ lang und $11 \mathrm{~g}$ schwer (s. Tafelfigur 1 und 2). Die Tube hat einen etwas kugeligen Tumor, der am uterinen Teil umfangreicher wird als am abdominalen. In der Mitte ist eine nicht allzatiefe Rinne. Das uterine Ende mit sichtbarem Tubenlumen ist deutlich za erkennen. Es läuft gerade und $1 \mathrm{~cm}$ weiter frei und geht dann in den geblähten Teil der Tube auf. Am anderen Pol der Tube sieht man das abdominale Ende mit deutlich erkennbaren Fimbrien und geöffnetem Ostium abdominale. Das Mittelstück ist der gravide Teil, es ist kolbig ver- 
dickt: Dicke 2,5 cm, Umfang $7 \mathrm{~cm}$. Auf der Höhe der am stärksten prominierenden Stelle findet sich eine explosionsartige Rautenform der Rupturöffnung, die $0,4-0,6 \mathrm{~cm}$ breit ist. Aus der Rupturstelle ragen die Blutcoagula hervor. Die Oberfläche der Tube ausserhalb der Rupturgegend ist glatt und frei von Adhäsion. Die subserösen Gefässe zeigen sich erweitert und haben das Aussehen von kleinen varizenartigen Gebilden.

Auf Grund des erwähnten Befundes ergibt sich ohne weiteres eine geborstene Isthmusschwangerschaft.

II. Die linke Seite der exstirpierten Adnexe. Das Ovarium ist cystisch degeneriert und 2 Finger gross; an ihm hängt die normal aussehẹde Tube.

\section{Die Vorbereitungen zur mikroskopischen Untersuchung.}

Das Präparat, das aus der rechten Tube stammt, wird nach der vorsichtig ausgeführten Härtung mit Alkohol und Xylol unter Paraffineinbettung in Serienschnitte zerlegt. Die Schnittdicke war $10 \mu$. Die Färbung wurde mit Hämatoxylin-Eosin und nach dem Verfahren van Gieson ausgeführt. Anfangs habe ich nur an der Rupturstelle 752 Präparate geschnitten, dann von den nicht im Bereich dieser Stelle liegenden Partien stellenweise 460 Präparate, weil es bei meinem Falle von grossem Interesse war, genau die Entwicklungsrichtung des Eies zu untersuchen.

\section{Besondere Beschreibung des mikroskopischen Befundes.}

I. Verschleppung der Zotten und Zottenelemente in die Venen.

An diesem Präparat habe ich erstens das gefunden, was auch Kiutsi gefunden hatte; die Verstopfung der abführenden Vene. In der Nähe des Eibettes und der Mesosalpinx finden sich Zotten und Zottenteilchen in die Venen verschleppt. Besonders stark aber tritt die Zottenmasse bei grösseren Venen hervor. Bei einigen Venen sind die Zottenelemente so dicht in das Lumen eingedrungen und die Venen so stark verdünnt, als ob sie direkt in den Muskeln steckten, wie es schon Leopold' ${ }^{1}$ ' beschrieben hat. Einige Venen sind an den betreffenden Stellen sehr gedehnt und verdünnt. Meist ist auch die Intima gedehnt, nur stellenweise ist sie mit Endothelzellen versehen. Dagegen habe ich einige Venen gefunden, die

1) Leopold, Dieses Arch. 1876. Bd. X. S. 262. 


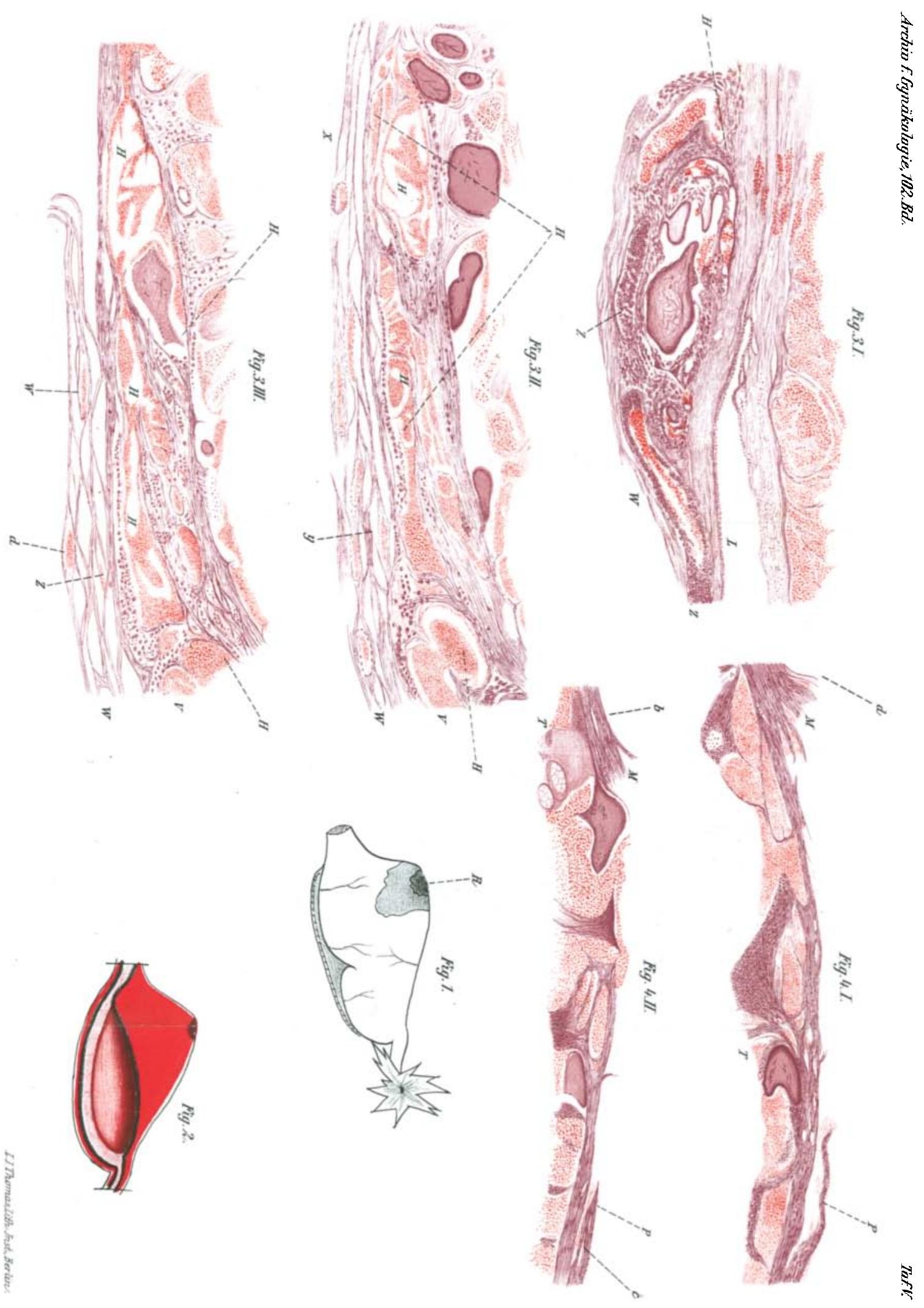


246 Taniguchi, Ueber Blutung bei der Ruptur der Tubenschwangerschaft.

nur mit Zotten und Zottenepithel vollständig oder unvollständig verstopft waren, und die Venenwand war meist hypertrophisch wegen des Schwangerschafteinflusses. Ferner habe ich eine Verdickung der Intima bemerkt. Diese grösseren Venen mit. Zottenverschleppung gehen alle vom intervillösen Raum aus, was man sehr leicht serienweise verfolgen kann.

Schon beim ersten Blick auf die Präparate fiel es mir auf, dass in den grösseren Venen reichliches Zottenmaterial lag und sie total verstopfte. Diese Verstopfung erstreckte sich über eine ziemlich lange Strecke der betreffenden. Vene. Ausserordentlich interessant war hierbei die Untersuchung mit Serienschnitten. Ich möchte daher hier die Darstellung von 3 Präparaten geben.

I. Präparat (Tafelfig. 3, I): Abschnitt mit vollständiger Zottenelementverstopfung und kleiner Venenruptur. Die Vene läuft quer und schräg. In der Nähe der Mesosalpinx wird sie von fötalen Elementen total verstopft. Neben den Zotten ist überhaupt gar kein Raum mehr für das flüssige Blut vorhanden. Je näher die Vene an den intervillösen Raum kommt, desto mehr erweitert sie sich. Die Vene ist hier mit Blutgehalt erfüllt, die Venenwand deutlich gedehnt. Die Wand der Vene ist demgemäss sehr dünn geworden, aber die Endothelien sind stellenweise noch zu sehen. Die Wand findet sich endlich nach. dem Eibett zu gerissen und bildet hier ein kleines Hämatom in der Venenumgebung. Die Wand ist nicht gleichmässig dick, sondern sie verdünnt sich ganz ungleichmässig. Diese ungleichmässige Dehnung rührt von den verschiedenen Graden des Widerstandes des umgebenden Gewebes gegen den aufsteigenden Druck des Blutes her.

II. Präparat (Tafelfig. 3, II): Abschnitt mit totaler Verstopfung durch Zottenelemente und mit grosser Venenruptur. Dieser Abschnitt liegt an der Ruptur der Tube und näher an der Mitte der Rupturstelle als im I. Präparat. Hier ist die Erweiterung der Vene stärker und grösser als dort. Der Abschnitt zeigt auch, dass die Vene wie mit einem Dilatator sehr stark gedehnt worden ist. Die Venenruptur und die Hämatome sind hier sehr dentlich erweitert. Die Wand der Vene ist sehr dünn geworden, und die Endothelzellen fehlen stellenweise. In der Tubenwand in der Nähe des Eibodens findet man auch einige kleine Hämatome, und man kann sofort serienweise die Verbindung mit dem Venenriss beobachten.

III. Präparat (Fig. 3, III): Abschnitt mit Venenruptur, Haematoma und Platzen nach den intervillösen Räumen. Hier ist diese 
Vene dieselbe wie im II. Präparat, und findet man ein Zerreissen des Hämatoms, das fern von dem Venenriss liegt, nach den intervillösen Räumen und nach der Eikammer zu. Hier findet man sofort sehr interessante Tatsachen. Einige intervillöse Räume sind in Verbindung mit der gerissenen Vene normal geblieben, während andere Teile der intervillösen Räume in Verbindung mit dem Hämatom gerissen sind. Eine Störung des Blatkreislaufs im intervillösen Raum ist daher nicht überall zustande gekommen, vielmehr ist der Zufluss von Blut aus den Arterien wie der Eintritt in die Venen so geblieben wie sonst. An einzelnen Stellen aber führt nicht die Vene das Blut aus dem intervillösen Raum, sondern zwischen Vene und ihm liegt ein Bluterguss im Gewebe. An einzelnen Stellen des intervillösen Raumes, an denen die Blutverhältnisse noch normal sind, fand ich trotzdem die Zeichen der Staung.

Warum sind die Hämatome in den intervillösen Räumen in Verbindung mit den geplatzten Venen von Anfang an nicht geplatzt? Das ist ganz begreiflich, weil der Druck in den betreffenden intervillösen Räumen stärker ist als in den anderen. Daher sind die Hämatome in den relativ druckniedrigeren intervillösen Räumen geplatzt.

An meinem Präparate habe ich das Einwachsen der Zotten oder Zottenelemente in die Arterie [Zedel'1), Fellner2)] nirgends beobachtet, wie Veit (l. c.), Füth (l. c.) u. a. es schon ausgesprochen haben.

II. Durch Staung ausgedehnte und aufsprungfertige Venen.

In der Tubenwand habe ich mehrere gestaute und aufsprungfertige Venen beobachten können. Einige gefüllte Venen sind am Rande des Eibettes riesig erweitert; die Venen in der tieferen Wandschicht sind auch in kolossale Stauung gesetzt. Vielfach sind sie mit Zotten uud Zottenteilchen gefüllt. Nach dem Peritoneum zu sieht man auch enorm mit Blut gefüllte Venen. Weiter bemerkt man dicht unterhalb des Peritoneums recht varixartige erweiterte Venenräume, die nach der Bauchhöhle zu hervorspringen. Die Wand ist gerade nur noch papierdünn - aufsprungfertige Vene. Aehnliches findet man auch in der Mitte der Tubenwand. Ferner habe ich die Verdickung der Intima in 2 grossen Venen gefunden, die

1) Zedel, Deutsche med. Wochenschr. 1895.

2) Fellner, Dieses Archiv. Bd. 74. S. 481. 
248 Taniguchi, Ueber Blutung bei der Ruptur der Tubenschwangerschaft.

eine Folge des Ueberdrucks, verursacht durch Stauung, ist. In meinem Falle sind die rerschiedenen Venen in kolossale Stauungen gesetzt worden, und so sind die Staungen in jeder Schicht der Fruchtkapsel entstanden. Diese hochgradigen Stauungen kann man natürlich verschiedenartig erklären: Es kann sich um eine Hemmung des venösen Abflusses bei ungestörtem arteriellen Zufluss oder um eine Blutüberfüllung bei normalem Abfluss handeln. Sind die Kollateralbahnen von vornherein ungenügend, so lassen sich diese Zustände natürlich viel leichter verstehen und zwar durch die vollständige oder unvollständige Zottenverschleppung in die kleinen oder grossen Venen.

\section{Zotten und Trophoblast.}

An meinem Präparat ist der Zustand der Zotten sehr verschieden. An einzelnen Zotten fand ich die venösen Gefässe der Zotten mit fötalem Blut prall gefüllt, so dass ich von einer Staung in den Zotten sprechen musste. In dem Blute fand ich fötale Erythroblasten $(7-9 \mu)$, Poikilozyten $(9 \mu)$, Megalozyten $(13 \mu)$ und freie Kerne $(2-3 \mu)$. Ferner konnte ich an einer Stelle fötale Blutungen nach dem intervillösen Raume, an einer anderen Anämie und Nekrose im Stroma beobachten. Die Zotten sind auseinandergedrängt, stellenweise aber sah ich die Zottenkompression. An meinem Präparate wird die langgestreckte Zotte im dichten Zottenkomplexe durch von beiden Seiten andrängende Zotten stark geklemmt. Infolgedessen wird die komprimierte Stelle völlig eng und schliesslich geknickt, so dass kein Blut mehr passieren kann. Merkwürdig aber ist, dass die beiden nicht komprimierten kolbigen Endteile der betreffenden Zotte ein kontrastierendes Bild darbieten. In der relativ dickeren Aufbauchung findet man eine Zottenapoplexie, in den anderen Endteilen aber eine Zottenischämie.

Also ich komme in Uebereinstimmung mit Kiutsi (l. c.) dazu, diese Beziehung zwischen Zottenkompression und Entstehungsweise der Zottenstauung und der Zottenblutung, sowie der Zottenanämie durch eine Kompression erklären zu können. Es ist ganz begreiflich, dass die Zotten durch den intervillösen gesteigerten Ueberdruck auseinandergedrängt werden.

Der Nitabuch'sche Fibrinstreifen ${ }^{1}$ ) an der Grenzschicht

1) Nitabuch, Beiträge zur Kenntnis der menschlichen Plazenta. Inaug.-Dissert. Bern 1899. 
zwischen dem fötalen Zellenlager und dem mütterlichen Gewebe hat in meinem Präparat einen erheblich undeutlichen Charakter gezeigt. Die geringe Hubrecht'sche periphere Trophoblastschale ${ }^{1}$ ), die aus fötalen Zellen und zwar den Abkömmlingen beider Zellschichten - Synzytium und Langhans'schen Zellen - besteht, um das Ei mit dem mütterlichen Gewebe sich verbinden zu lassen, geht ohne förmliche fibrinoide Grenzschicht nur diffus in das mütterliche Gewebe über. In meinem Falle sehe ich den echt ausgedehnten fibrinoiden Untergang der innersten Eikammerwand. Auch habe ich stellenweise den ebenso gleichen Untergang des Trophoblastes beobachtet. Sie scheint erst plasmoidal und zugleich fibrinoid verändert gewesen zu sein, da man an einigen relativ gesunden Stellen noch etliche Synzytiumsprossen von ihr in den intervillösen Raum springen sehen kann. Das ist ein Einfluss, der von einem Ueberdruck im intervillösen Raum herrührt. Dass durch einfache Stagnation im Zwischenzottenraum schon die Deziduainseln auch sekundär geschädigt werden können, hat $\mathrm{Bumm}^{2}$ ) beim weissen Infarkt nachgewiesen.

\section{Sonstige Blutung.}

Blutansammlung, welche die Zotten auseinandergedrängt hatte, fand ich im intervillösen Raum, Blutung auch im Tubenlumen, und endlich lagen in der Wand der Tube Hämatome, die dem Peritonealïberzug sehr nahe waren, und. welche den Anschein erweckten, als ob sie in die Bauchhöhle durchbrechen wollten.

Gegen das Bindegewebe zeigt die Blutung keine scharfe Begrenzung. Untersuchen wir die Hämatome serienweise, so werden wir zuletzt meist an eine Vene kommen, die mit Zotten und Zottenepithelien verstopft ist. Und so glaube ich, dass man eine solche Blutansammlung im Gewebe fast als eine wandlose erweiterte Vene ansehen darf, die sich auch im Gewebe als geplatzt darstellen kann. So kann man an meinem Präparat die mehr umschriebenen Hämatome fast ausnahmslos nach Venen hin verfolgen.

\section{Ruptur.}

A. Rupturstelle.

Was den Ort betrifft, in welchem am häufigsten die plötzlichen Rupturen der schwangeren Tube vor sich gehen, so gibt es dar-

1) Vgl. Zentralbl. f. allgem. Pathol. usw. 1901. S. 467.

2) Bumm, Grundriss der Geburtshilfe. 8.Aufl. 1912. S.400. Wiesbaden. 
über von vielen Autoren sich widersprechende Angaben. Einige führen Fälle von Ruptur an der Plazentarstelle an [Conrad und Langhans ${ }^{1}$, Ulesko-Stroganowa (l. e.)] oder in nächster Nähe der Plazentarstelle [Martin und Orthmann²)], andere [Kreisch $\left.{ }^{3}\right)$ ] an der ihr gegenüberliegenden Seite als der am meisten verdünnten. Aschoff ${ }^{4}$ ) hat in seinem ausgezeichneten Referate über die Anatomie der Aetiologie der Tubenschwangerschaft folgendes geschrieben: "Die Rupturstelle schwangerer Tuben aus dem ersten Monate sitzt ausschliesslich an der Plazentarstelle". Es kann bei einem Riss der Tube an der Plazentarinsertionsstelle die Blutung dadurch stehen, dass die Plazenta die Rissstelle direkt verstopft, wie Schoemacher ${ }^{5}$ ) in einem Fall bemerkt zu haben glaubt. An meinem Präparat findet man die Rissstelle an der der Plazentarstelle gegenüberliegenden Seite.

An der Rupturstelle sind der intervillöse Raum und mehrere Kapillaren bzw. zwei kleinste Gefässe eröffnet. Das eine Gefäss ist aber erweitert und stellenweise sind Endothelien vorhanden. Das andere ist nicht erweitert, die Endothelien sind normal erhalten. Es scheint auf den ersten Blick, dass ersteres eine Vene und letzteres eine Arterie ist; wenn man aber die Bilder serienweise verfolgt, so tritt es sofort deutlich zu Tage, dass die zwei Gefässe Venen sind. Die erweiterte zeigt eine Verschleppung der Zottenelemente, die andere hingegen tritt in ein Hämatom im Gewebe ein und zerreisst hier die Wand derselben. Dieses Hämatom stammt aus einer anderen (dritten) Vene mit Zottenverschleppung.

\section{B. Form der Rupturöffnung.}

Die Oeffnung, die ich bei dem makroskopischen Befunde beschrieben habe, zeigt eine explosionsartige Rautenform (Tafelfigur 1,2), was wohl eine Folge der plötzlichen Drucksteigerung ist. Während die Form der Rupturöffnung bis heute von Autoren verschiedenartig beschrieben ist, glaube ich die Anhaltspunkte für eine einheitliche Darstellung geben $z u$ können. Bei der Ruptur

1) Conrad u. Langhans, Dieses Archiv. 1876. Bd. 9. S. 337.

2) Martin u. Orthmann, in Martin, Die Krankheiten der Eileiter. 1895. S. 334. Leipzig.

3) Kroisch, Monatsschr. f. Geb. u. Gyn. 1899. Bd. 9. S. 794.

4) Aschoff, Zentralbl. f. allgem. Pathol. u. pathol. Anat. 1901. S. 487.

5) Schoemacher, Zentralbl. f. Gyn. 1899. S. 91. 
ist natürlich die Form der Oeffnung etwas anders gestaltet wie bei der Usur, und sie muss es. Die Form des durch Usur entstandenen allmählichen Durchscheinens des Eies stellt sich meist in kreisrunder Gestalt dar, ihre Ränder sind nach "aussen" umgelegt. Vgl. Werth (l. c.).

C. Ränder der Rupturstelle.

Ich habe an der Rupturstelle nirgendswo den aggressiven Vorgang seitens der fötalen Zottenelemente finden können. An den Rändern der Ruptur findet man deutlich die Verdünnung der Tubenwand (bis 4,4 mit Mess-Okular 3 und Objektiv D. D. $=4,4 \times$ $3,8=16,72 \mu)$, die Auflockerung durch die ödematöse Auftränkung, Deportation der Zottenelemente, die Veränderung der PeritonealEpithelzellen der Tubenwand und hämorrhagische Infiltration und zwar die beiden letzteren hier stärker als anderswo. Ein grösseres Gefässlumen in der Nähe der Ruptur fehlt vollständig. Dagegen habe ich Blutlakunen in fibrinösen Massen und in auseinandergedrängtem Gewebe der Tubenwand gefunden. Ferner habe ich auch den Trophoblast gesehen und zwar hier den fibrinoiden Untergang desselben. Die Rupturränder sind in einer kleinen Strecke etwas nach aussen umgelegt (Tafelfig. 4, I n. II). Man kann wohl das Verhältnis derselben durch die Figur verstehen. An der Tafelfigur 4, I, findet sich nur die Zerreissung der peritonealen Epithelschicht und des äusseren Teiles der mittleren Schicht der Tubenwand, während der innere Teil derselben noch nicht gerissen ist. Diese Tatsache spricht gegen die aggressiven Vorgänge der Zottenelemente, und dieser Befund spielt für den Ueberdruck in der Fruchtkapsel eine sehr grosse Rolle. Die Ränder haben sich als Folge der Zerreissung der äusseren Schicht, die nur allein gerissen ist, der Tubenwand und durch den Druck von Eiresten sowie koaguliertem Blut nach aussen umgelegt.

Kiutsi betont: "Dieses Ausbleiben der fötalen Zellen an beiden Rändern muss für die Ursache der Ruptur sehr bedeutungsvoll sein, der Befund spricht sehr dagegen, dass man als Ursache der Tubenruptur die auffressende Energie des Trophoblastes in Anspruch nehmen könnte." In dem Punkte, dass die deutliche Verdünnung der Wand als prädisponierte Partie für die Rupturstelle angenommen und die auffressende Energie seitens der fötalen Elemente als Ursache der Tubenruptur nicht anerkannt werden kann, stimme ich völlig mit Kiutsi (l. c.) überein, nur fand ich an 
252 Taniguchi, Ueber Blutung bei der Ruptur der Tubenschwangerschaft.

meinem Präparate fötale Zellen auch an den Rändern der Ruptur. Nach meiner Meinung muss man als disponierte Stelle die dünnste ansehen, die am stärksten hämorrhagische Inflitration und Aenderung der peritonealen Epithelzellen zeigt.

\section{Tubenwand.}

\section{A. Peritonealepithel der Tubenwand.}

An meinem Präparate findet man die Veränderungen der Peritonealepithelzellen. Die Epithelzellen zeigen sich an einzelnen Stellen der Tubenoberfläche, je näher an der Rupturstelle, um so stärker erhöht, meist so stark, dass dieselbe zylindrische Gestalt erhalten haben. Solche Strecken liegen zum Teil unter dem Schutz von Blutanhaftungen, doch kann man auch gelegentlich ohne solche Bedeckung eine Erhöhung des Peritonealepithels beobachten. Hier und da findet sich an der Oberfläche der Tubenwand Peritonealepithel von mehreren Schichten und isolierte sich von der oberflächlichen Schicht ablösende Epithelzellen, die in das gelockerte Wandgewebe eindringen. Ich habe in der peritonealen Umhüllung den Deziduazellen ähnliche Elemente beobachtet, die Walker ${ }^{1}$ ) und $Z_{w}$ eifel ${ }^{2}$ ) schon beschrieben haben. Diese Zellen gehen aus den Bindegewebszellen der Serosa hervor, weil man an der Oberfläche stellenweise normale Peritonealzellen nachweisen kann. Die von Walker diesem Befunde gegebene Deutung als eines Beweises für die primär peritoneale Lokalisation des Eisitzes durch den von Schmorl ${ }^{3}$ ) und Kinoshita ${ }^{4}$ ) gefürten Nachweis, dass auch bei uteriner Schwangerschaft die Entwicklung eines solchen grosszelligen Gewebes im Beckenbauchfell und Eierstocksstroma nicht selten ist, ist hinfällịg geworden.

\section{B. Mittlere Schicht der Tubenwand.}

Bezüglich der Tubenwand ist zu bemerken, dass deren Dicke in den verschiedenen Abschnitten der Tube ausserordentlich wechselt. In der Nähe des Eibodens kann man eine deutliche Verdickung der Tube beobachten. Dies rührt zum Teil von Hyperämie dés Gewebes, zum Teil von Oedem und Infiltration der Wand her. Be-

1) Walker, Virchow's Arch. Bd. 107. S. 72.

2) Zweifel, Klinische Gynäkologie. 1892. S. 252.

3) Schmorl, Monatsschr. f. Geburth. a. Gyn. 1897. Bd. 5. S. 46.

4) Kinoshita, Monatsschr. f. Goburtsh. u. Gyn. 1898. Bd. 8. S. 500. 
sonders konnte ich die hochgradige Auflockerung der Gewebe durch ödematöse Durchtränkung beobachten. Die Auflockerung erstreckte sich nicht nur bis in die Nähe des Eibodens, sondern auch bis in eine weitere Zone und bis zur Rupturstelle, aber sie ist schwächer, ausser in der Nähe des Eibodens. Das Oedem ist ebenso wie bei der Uterinsehwangerschaft. Man findet zwischen den Bindegewebsund Muskelfasern die Transsudation und Exsudation, sodass die Fasern nunmehr sehr deutlich erscheinen. Ferner habe ich hier und da die Muskelbündel durch hyalines Bindegewebe und die zweifellos wirkliche Muskelhypertrophie, die wohl in der Nachbarschaft des Eisitzes am stärksten ist, gesehen.

Zu dem bereits. Gesagten füge ich noch zu, dass ich zahlreiche fötale Zellen, die sog. Veit'sche Deportation des Chorionepithels in der Tubenwand und in den Venen, besonders in der Nähe des Eibodens gefunden habe. Ferner habe ich gestaute, aufsprungfertige und solche Venen, deren Intima deutlich. verdickt war, sowie hämorrhagische Infiltration in der Wand beobachten können. Alle diese Prozesse sind in der Nähe des Eibodens recht deutlich zu sehen.

Cornil ${ }^{1}$ ), Webster ${ }^{2}$, Monol ${ }^{3}$ ) u. a. glaubten eine Wucherung der Gefässwandzellen speziell der Endothelien annehmen zu dürfen. Schambacher ${ }^{4}$ ) nimmt an, dass die deziduaähnlichen Zellen ihren Ursprung in der Gefässwand der Bindegewebszellen haben.

Martin ${ }^{5}$ ) und Aschoff (1. c.) konnten in vielen schwangeren Tuben nichts von einer Entzündung nachweisen, ein Umstand, der beweist, dass wenigstens beträchtliche Leukozyteninfiltration nicht als eine Folge der Gravidität angesehen werden kann. Weiterhin scheint die Beweisführung von Dührssen ${ }^{6}$ ), Engström ${ }^{7}$ ) und Petersen ${ }^{8}$ ) durchaus glaubhaft und richtig zu sein, dass die gefundene Entzündung nicht vom $\mathrm{Ei}$ herrühren kann, da man eine solche nicht gleichmässig zu beiden Seiten des Eisitzes vorgefunden

1) Cornil, s. Veit, Ueber Extrauteringravidität. S. 24.

2) Webster. Deutsch von A. Fiermann, Berlin 1896. Karger.

3) Monol, s. Schambacher, Zeitschr. f. Geb. u. Gyn. Bd. 48. S. 434.

4) Schambacher, Zeitschr. f. Geb. u. Gyn. 1903. Bd. 48. S. 428.

5) Martin, Die Krankheiten der Eileiter. Leipzig 1895.

6) Dührssen, Dieses Arch. 1892. Bd. 54. S. 207.

7) Engström, Mitteil. a. d. gyn. Klinik. 1898. Bd. 2. S. 383. - Zeitschrift f. Geb. u. Gyn. 1896. Bd. 38. S. 122.

8) Petersen, Beitr z. pathol. Anat. d. graviden Tube. Berlin 1902. 
254 Taniguchi, Ueber Blutung bei der Ruptur der Tubenschwangerschaft.

hat. Vielmehr zeigt sich regelmässig eine Zunahme nach dem Uterus hin. Oft trifft man sie nur uterinwärts vom Ei, auf das Fransenende zu garnicht. An meinem Präparat habe ich deutliche Leukozyteninfiltration in der uterinen Seite serienweise gefunden.

\section{Schleimhaut der Tubenwand.}

An meinem Präparat ist das Aussehen des Tubenlumens makroskopisch sehr verschieden (T'extfig. 5, 1, II). Das Tubenlumen erweitert sich, je näher es an das äussere Ende herankommt, mehr

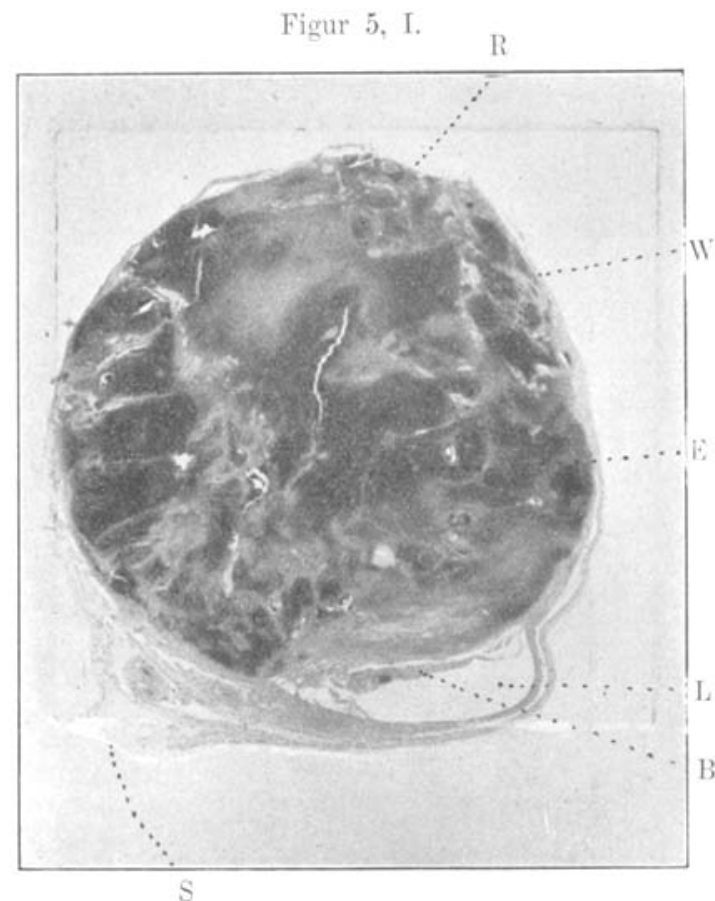

Abschnitt in der Rupturstelle der schwangeren Tube. Die Eikammer (E) liegt in der Tubenwand (W). Das Tubenlumen (L) ist ziemlich erweitert. In ihm findet sich koaguliertes Blut (B). $\mathrm{R}=$ Rupturstelle. $\mathrm{S}=$ Stiel.

und mehr und wird nach einer Seite gedrängt. An einem grossen Teil der hervorragenden Fruchtkapsel fehlen EpitheI, Muskelfasern und Gefässe. Dagegen finden sich fibröses Bindegewebe, Blutkörperchen, deziduaähnliche Zellen, Zotten und Zottenepithel - Reflexa -. v. Both ${ }^{1}$ ) und Kroemer ${ }^{2}$ ) baben deutlich ausgesprochene

1) v. Both, Monatsschr. f. Geb. u. Gyn. 1899. Bd. 9. S. 782.

2) Kroemer, Dieses Archiv. 1903. Bd. 48. H. 1. S. 57. 
deziduale Veränderungen in der Tube beschrieben. Webster ${ }^{1}$ ) hat die Decidua vera tubaria für eine ganz regelmässige Bildung gehalten. Auf Grund meiner Präparate muss ich die Webster'sche Theorie, dass eine deziduale Umwandlung der Tubenschleimhaut das Haften des Eies überhaupt nur ermöglichte, verneinen. Veit (l. c.) hat darüber folgendes gesagt: „So kann man also die Ausbildung einer echten Decidua vera bei Tubenschwangerschaft als sehr seltene Ausnahme zugeben, dass Veränderungen in der Tube deutlich sind, die man wohl in unbeschränktem Sinne als deziduaähnliche be-

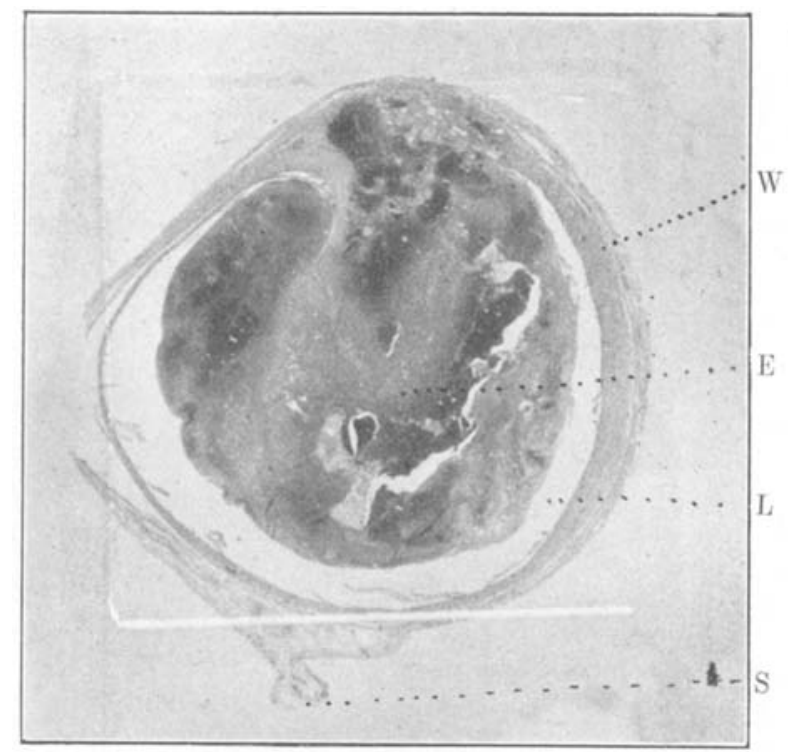

Abschnitt in der Nähe der Ampulla tubae. Die Eikammer (E) ragt in das Tubenlumen (L) hinein. Dieses ist deutlich erweitert, ein grosser Teil der Eikammer ist von ilhm umbillt. $W=$ Tubenwand. $S=$ Stiel.

schreiben kann". Franz²) erklärt, dass die Reftexa aus hyalinem und fibrösem Gewebe mit deziduaähnlichen Zellen bestehe. Das Tubenepithel, aus dem sich, wie einige Autoren, Hofmeier ${ }^{3}$, Gunsel ${ }^{4}$ ) annehmen, das Synzytium bilde, habe ich an meinen

1) Webster, Die elitopisehe Schwangerschaft. Deutsch von Eiermann. Berlin 1896.

2) Franz, Verhandl. der deutschen Gesellsch. f. Gyn. 10. Kongr. 1903. S. 256 .

3) Hofmeier, Zeitschr. f. Geb. u. Gyn. 1896. Bd. 35. S. 414.

4) Gussel, Zentralbl. f. allgem. Pathol. 1891. S. 6. 
256 Taniguchi, Ueber Blutung bei der Ruptur der Tubenschwangerschaft.

Präparaten nicht gefunden. Weil ich aber einige Faltenverwachsungen und Entzündungszeichen (vgl. obigen Befund) beobachtet habe, glaube ich, dass die Patientin früher Salpingitis durchgemacht hat.

\section{Der Sitz des Eies.}

In meinem Präparate sitzt das Ei zum Teil in dem Tubenlumen und zum Teil in dem Bindegewebe und der Muskelschicht der Tubenwand (Tafelfig. 2 und Textfig. 5, I, II). In meinem Falle scheint mir die Eilage sehr wahrscheinlich durch Annahme der Bindegewebshöhle dicht unter dem Tubenkanal, wie es schon Veit (l. c.) gesagt hat, erklärt zu sein, wenn ich den Eieinbettungsmodus von Graf Spee ${ }^{1}$ ) annehme, dass nämlich das Ei durch die Epithellücke subepithelial sein Bett findet. Der uterinale Pol des Eies drängt sich nach der uterinen Seite und nach der Oberfläche der Tube und zwischen die zirkuläre und longitudinale Muskulatur hinein, während der ampullare Pol nach der ampullaren Seite und dem Hohlraum der Tube eingedrungen ist. Das Ei liegt daher schief in der Tube. Die Wand der Eikammer ist reichlich mit Trophoblast besetzt, das wohl mit der Deziduaspaltung [Hofmeier $\left.{ }^{2}\right)$ in das mütterliche Gewebe hineingelangt, d. h. durch die erweiterten Lymphspalten [Veit $\left.\left.{ }^{3}\right)\right]$ hineinbefördert wird. Um die Eihöhle herum, d. h. innerhalb der Tubenwand mit Ausnahme der Filage findet sich Trophoblast von verschiedener Dicke, oder es fehlt auch ganz. Die ganze Eilage erstreckt sich von der Anfangspartie der Pars isthmica bis zur Pars ampullaris. Während die Eikammer an dem inneren Ende das Tubenlumen etwas umgibt, ist es an dem äusseren gerade umgekehrt, wo das Lumen die Eikammer umhüllt. Ich kann, mich stützend auf mein Präparat, es aussprechen, dass bei der utero-tubaren Schwangerschaft das $\mathrm{Ei}$ sich in einer dicht am Uebergange des Isthmus in den Uterinteil der Tube gelegenen Stelle eingenistet und. sich dann nach der Uterinseite entwickelt hat. Deswegen handelt es sich anstatt der uterotubaren Schwangerschaft sehr wahrscheinlich um isthmische, wie Werth ${ }^{4}$ ) schon gesagt hat.

1) Graf Spees. Kollmann, Handatlas der Fntwicklungsgeschichte des Menschen. 1907. Bd. 1. Fig. 144 u. 145. Jena.

2) Hofmeier, Zeitschr. f. Geb. u. Gyn. 1896. Bd. 35. S. 414.

3) Veit, Zottendeportation.

4) Werth, Winckel's Handbuch. Bd. 2. T. 2. S. 741. 
Der Konfigurationsmodus Kussmaul's ${ }^{1}$ ) und Kiutsi's ${ }^{2}$ ) bei geborstener Isthmusschwangerschaft ist wenigstens in meinem Falle nicht zu finden. In meinem Fall sitzt das $\mathrm{Ei}$ nicht "intratubar" oder in beschränktem Sinne "paratubar", sondern "gemiseht intraet paratubar" (Tafelfig. 2).

\section{Woher stammt die Blutung, aus der die Graviditas tubaria sich beinahe verblutet hat?}

Untersuche ich die Herkunft der Blutung, aus der sich eine Frau bei Ruptur der Tubenschwangerschaft verbluten kann, so will ich diese Frage nur an der Hand meines Präparates beantworten. Von Wichtigkeit scheint mir dabei zu sein, dass hier der intervillöse Raum sich in die Bauchhöhle bei der Ruptur eröffnet hat. An einzelnen Stellen waren die Zotten, wie erwähnt, durch den Bluterguss weit auseinandergedrängt. Ich konnte nachweisen, dass dies ebenso wie in dem Fall von Kiutsi dadurch sich erklärt, dass eine Vene mit Zotten verstopft und gerissen war. So ist die Annahme berechtigt. dass das Blut in den intervillösen Raum hineinströmen, aber nicht aús ihm herauskommen konnte. Der Ueberdruck, der dadurch entstand, zerriss zuerst die Vene und dann die Wand der Tube. In den intervillösen Raum hinein entleerten sich dann die Venen und gleichfalls ein oder das andere Hämatom, welches sich schon vorher gebildet hatte. Mit Sicherheit kann ich nach meinem Präparate sagen, dass hier ein grosser Teil der Blutung aus dem intervillösen Raume stammte.

Die Ursache dafür ist ja ganz klar. In gleicher Weise wie Waldeyer ${ }^{3}$ ) den Blutgehalt des intervillösen Raumes nachweist und $B u m m^{4}$ ) den Eintritt der Arterien and den Austritt der Venen schildert, tritt auch bei Tubenschwangerschaft aus arteriellen Oeffnungen Blut zwischen die Zotten. Die Zahl der abführenden Venen ist auch hier verhältnismässig gering. Die Tubenarterie stammt aus der A. uterina und der A. ovarica. Der Ramus tubae beider verbindet sie ${ }^{5}$ ). Die Rissmündungen der intervillösen Räume liegen ganz in der Nähe der ursprünglichen Stammarterie. Deswegen ist

1) Kussmanl, Winckel's Handbuch. Bd. 2. T. 2. S. 670 .

2) Kiutsi, Dieses Archiv. Bd. 95. S. 500.

3) Waldeyer s. Veit, Zottendeportation. S. 53.

4) Bumm, Dieses Archiv. 1890. Bd. 37. S. 1.

5) Rauber-Kopsch, Lehrbuch der Anatomie. 7. Aufl. 1899. Abt. 3. 
258 Taniguchi, Ueber Blutung bei der Ruptur der Tubenschwangerschaft.

die Geschwindigkeitsverminderung durch die Reibung verhältnismässig gering. Mit dem gewöhnlichen Blutdruck und der gewöhnlichen Schnelljgkeit strömt das Blut aus den arteriellen Kapillaren in den intervillösen Raum; in ihm verlangsamt sich die Geschwindigkeit. Je grösser nun das deziduale Polster ist, um so geringfügiger werden der Druck und die Geschwindigkeit sein, mit denen das Blut in den intervillösen Raum eintritt. Da bei Tubenschwangerschaft mangelhafte deziduale Polster vorkommen, so dass das Ei direkt in die Tubenwand - Bindegewebe und Muskel zu liegen kommt, zeigen sich hier ungenügende kapillare Bildungen. Wer sie mit den Kapillaren der Uterusdezidua vergleicht, findet einen grossen Unterschied. Bei dieser mangelhaften dezidualen Anlage kann hier keine Seitenarterie zu einer bedeutenden Vermehrung des genannten Querschnittes beitragen. Nun, da die Geschwindigkeit des Blutes gleich dem in einer Sekunde durchflossenen Volumen dividiert durch den Querschnitt ist, so ergibt sich, dass die Stromgeschwindigkeit im Eibette bei der Tubargravidität grösser sein muss als bei der uterinen Schwangerschaft. Ebenso dürfte der Triebkraftverbrauch, der näher in den kleinsten Arterien und Kapillaren stattfinden soll, wo der Reibungswiderstand besonders gross ist, geringer sein als beim Uterus. So hat die ungenügende Gesamtquerschnittsvermehrung der Arterien verursacht, dass bei den Arterien im tubaren Eibette keine bedeutende physiologische Minderung der Geschwindigkeit und der Triebkraft erfolgte.

Auf Grund dieser Erwägungen kann ich leicht erklären, dass die foudroyante Blutung bei der Ruptur der schwangeren Tube aus den geplatzten intervillösen Räumen stammt. Da also das Blut aus diesem Raume stammt, so strömt es aus den Arterien in die Bauchhöhle hinein.

Fragt man nach weiteren Stellen, aus denen die Blutung stammen kann, so muss ich betonen, dass an meinem Präparate grössere Arterien oder Venen an der Rissstelle nicht mitgerissen sind. Eine Blutung aus einer zerrissenen Arterie kann ich für meinen Fall wenigstens nicht finden.

Was die Venen betrifft, so ist ja ganz deutlich, dass die mit Zotten verstopfte Vene zwischen der Verstopfung und dem intervillösen Raum geplatzt ist. Aber wenn aus dem intervillösen Raum das Blut ohne weiteres in die Bauchböhle fliesst, so kommt äberhaupt kein Blut mehr in die Venen hinein.

Allerdings habe ich an der Rupturstelle verschiedene kleine 
Venen zerrissen gefunden. Es kann natürlich aus ihnen das Blut herauslaufen, aber die Hauptursache für die foudroyante Blutung kann ich in der Vene nicht finden. Für die Blutung bei dem Platzen der von mir untersuchten Tubenschwangerschaft spielt ebenfalls der intervillöse Raum die grösste Rolle. Die Venen sind von geringerer Bedeutung.

\section{Tritt die Blutung yor der Tubenruptur oder als Folge derselben auf?}

An meinem Präparat konnte ich durch Stauung ausgedehnte und aufsprungfertige Venen in der Tubenwand beobachten. Wenn man dem Verlauf der Venen folgt, so findet man sofort eine Verstopfung durch die Zotten oder Zottenelemente. Auch habe jch überall in der Tubenwand die Hämatome in Verbindung mit der Zottenverschleppung der Venen gefunden. Weiter habe ich eine interessante Vene in der Nähe der Mesosalpinx beobachten können, welche mit Zottenelementen total verstopft war. Sie ist, je mehr sie von der Thrombose der Zotten entfernt ist, desto mehr, ja bis zum Springen durch Stauung erweitert worden. Die Venenwand findet man ausserordentlich dünn, und stellenweise fehlen die Endothelien, an der inneren Seite sind sie kaum zu sehen. Die Vene hat endlich einen Riss auch nach dem Gewebe in der Innenseite der betreffenden Vene zu bekommen und hier ein Hämatom gebildet, aus dem dann Blut nach dem intervillösen Raum und der Eikammer strömt. Infolgedessen entstand ein plötzlicher Ueberdruck in dem intervillösen Raum und der Eikammer, der die Ruptur der schwangeren Tube verursachte. Wenn die Verstopfung durch Zottenelemente nach der Ruptur eintritt, dann muss die Erweiterung durch Stauung und das Platzen der Venen fehlen. Die Ursache der durch Stauung ausgedehnten und aufsprungfertigen Venen sowie das Vorhandensein der Hämatome in der Tubenwand kann man ebenfalls leicht erklären.

Auf Grund dieser Erwägungen kann ich es bestimmen, dass die Blutung aus den Venen wit totaler Zottenverstoplung vor der Ruptur der schwangeren Tube eingetreten sein muss.

Die Blutung aus den intervillösen Räumen und den Venen an der 'Tubenrupturstelle kann ich folgendermassen erklären: Da die intervillösen Räume infolge der Zottenverschleppung der Venen durch die plötzliche Drucksteigerung hochgradig ausgedehnt wurden, sind einzelne intervillöse Räume durch das Eindringen des 
Blutes aus dem Hämatom geplatzt. Andere intervillöse Räume sind durch die plötzliche Druckverminderung bei der Ruptur der schwangeren Tube geplatzt. Die Blutung aus den geplatzten intervillösen Räumen nach der Bauchhöhle findet dauernd statt. Das Platzen der Venen an der Rupturstelle ist eine Folge der Tubenruptur, weil die Vene nicht erweitert und die Endothelzellen derselben normal erhalten worden sind. Infolge dieses Ergebnisses kann ich behaupten, dass die Blutung aus den intervillösen Räumen und den Venen an der Rupturstelle zweifellos nach der Ruptur der schwangeren Tube aufgetreten sein muss.

\section{Was ist die Herkunft der Blutung bei der Tubenruptur?}

Wenn die Blutung durch Torsion der schwangeren Tube entstehen kann, ist dies in meinem Falle nicht zu sehen. Als Hauptursache käme die Kontraktion in Betracht. Obwohl sie ohne weiteres die erhebliche Druckschwankung in dem intervillösen Raum veranlassen und das Zerreissen der Gefässwände zur Folge haben kann, weil die Gefässwand bei der Tubenschwangerschaft schwächer ist als bei der Uterinschwangerschaft, so spricht dennoch die Staung und das Zerreissen der Vene in meinem Falle gegen die Kontraktion. Zottenverschleppung in die Venen erklärt die Blutung hier viel lejchter. Bei meinem Präparat ist die Verschleppung der Zotten und Zottenelemente nicht in die Hauptvene, sondern in einen der grossen Aeste derselben erfolgt. Es könnte hier als Einwand eingeworfen werden, dass eine Kompensation ausserhalb des Gebietes der durch Zottenverschleppung verstopften Vene durch Kollateralbahnen bewirkt worden ist, wenn die Deportation nicht in die Hauptvene vor sich gegangen ist. Die kollateralen Bahnen aber sind, wie bekannt, bei der Tubenschwangerschaft weit weniger vorhanden als bei der Uterinschwangerschaft, und zwar sind alle grossen Venenäste in der Umgebung der genannten Venen mit Zotten oder Zottenelementen verstopft, obgleich die Thrombose der Zotten nicht vollständig ist. Daher tritt die Kompensationsstörung in den betreffenden Gebieten ein. Während wir in den Venen mit unvollständiger Verstopfung durch Zotten die Venenruptur nicht gefunden haben, lsönnen wir diese in den Venen mit totaler Verstopfung wohl beobachten. Diese Tatsache ist sehr wichtig. Wenn die Kontraktion die primäre Ursache der Tubenruptur wäre, dann müsste in all' den Venen ein Zerreissen eintreten. Hieraus kann man nun schliessen, dass die Blutung aus 
Taniguchi, Ueber Blutung bei der Ruptur der Tubenschwangerschaft. 261

den Venen durch Zottendeportation hervorgerufen ist. Die Kontraktion ist nur eine Nebenursache.

Wir möchten noch die Frage beantworten, waram ein so häufiges Abgerissenwerden der Zotten bei der Tubenschwangerschaft überhaupt vorkommt. Die letzten Ursachen will ich im folgenden darlegen.

Als erste käme die Kontraktion in Betracht. Dieses Moment kommt öfter bei der Tubenschwangerschaft als bei der Uterinschwangerschaft vor. Sie ruft ohne weiteres die erhebliche Druckschwankung in dem intervillösen Raum hervor und bringt weiter die Zotten zum Zerreissen und bewirkt die Verbindung der Zotten zur Abtrennung.

Die zweite Ursache gibt uns die anatomische Beschaffenheit der Tube, die man durch Vergleichung mit dem Uterus leicht begreifen kann. Die Tube hängt durch eine kleine Verbindung an dem Uterus, an dem Becken durch ein dünnes Ligamentum latum. Daher ist sie leicht beweglich und wird stets durch intraabdominale, auf sie einwirkende Reize aus ihrer Umgebung bewegt. Manchmal sieht man bei der Tubenschwangerschaft auch die Torsion. Bei Untersuchungen, beim Koitus usw. wird ebenfalls die Tube bewegt. Begreiflicherweise verursachen diese genannten lirscheinungen das Zerreissen der Zottenglieder; besonders bei der Torsion ist dies voll und ganz zu verstehen.

Während am Uterus das Eindringen der Zotten infolge der Dicke der Dezidua zu einer Vergrösserung des intervillösen Raumes führt, indem die Venen sich in ihm umwandeln können, liegen die Verhältnisse bei der Tubenschwangerschaft ungünstiger. Gelangen hier Zotten in die Venen hinein, so werden sie weit fortgeführt und können dann bei der Kontraktion abgerissen werden. Sie führen aber recht regelmässig zu einer Vergrösserung des intervillösen Raumes.

Als prädisponierende Ursache des Abgerissenwerdens der Zotten bei der Tubenschwangerschaft kann man die lose Zottenorganisation, das überzahlreiche Auftreten der Zotten, sowie die direkte Verbindung der grossen Venen mit dem intervillösen Raum ansehen.

Auf Grund obiger Betrachtungen möchte ich folgende Sätze aufstellen:

1. In meinem Falle ist die Rupturstelle der schwangeren Tube nicht an der Plazentarstelle, sondern an der ihr gegenüberliegenden 
Seite gelegen. Es ist die ganze Tubenwand in der Umgebung der Ruptur blutig suffundiert. So komme ich in Uebereinstimmung mit Kiutsi zu der Annahme, dass hier Staung vorliegt, und zwar dass die Stauung zur Ruptur geführt hat.

2. Als prädisponierte Stelle für die Ruptur sehe ich die dünnste an, die am hochgradigsten hämorrhagisch infiltrierte, welche auch eine Veränderung der Peritoneal-Epithelzellen zeigt. An diesem Präparat habe ich nirgendwo den aggressiven Vorgang seitens der fötalen Zottenelemente beobachtet.

3. Das kausale Moment der Tubenruptur ist der plötzliche Ueberdruck im Fruchtkapselraum dureh die Blutung aus den Venen mit totaler Zottenverstopfung, wie es Veit und Kiutsi betont haben.

4. Das Zottenabreissen und die Zottenverstopfung werden durch die Kontraktion verursacht. In meinem Falle habe ich nirgends Zotten in den Arterien gefunden. Wir können hier nur annehmen, dass die abgerissenen Zotten vom mütterlichen Blute fortgespült wurden.

5. Die foudroyante Blatung bei der Tubenruptur stammt aus den intervillösen Räumen. Dies ist eine Folge der direkten Fröfnung der Arterien in die intervillösen Räume, ihrer ungenügenden Gesamtquerschnittsvermehrung im Eibett und der geringeren Geschwindigkeitsverminderung durch Reibung infolge ihres kurzen Verlaufes von den ursprünglichen Stammarterien aus.

\section{Erklärung der Abbildnngen auf Tafel V.}

Figur 1. Natürliche Grösse der rechtsseitigen Isthmusschwangerschaft mit explosionsartiger Rautenform der Rupturöffnung (R). Von der Seite gesehen.

Figur 2. Sehematisches Bild, ohne vordere Peritonealoberfläche.

Schwarz $=$ Oberfläche der Tube.

Rot $=$ Mittlere Schicht der Tubenwand.

Violett $=$ Tubenschleimhaut.

Braunrot $=$ Eikammer.

Figur 3. Serie der Venenverstopfung mit Zottenelementen, der Venenruptur, des Hämatoms und des Platzens der Hämatomwand nach dem intervillösen Raum. Mikroskopische Zeichnung.

I. Abschnitt an der Tubenrupturstelle. Vollständige Zottenelementverstopfung $(\mathrm{Z})$. Venenruptur und kleines Hämatom $(\mathrm{H})$. Tubenlumen $(\mathrm{L})$. Tubenwand (W). 
Taniguchi, Ueber Blutung bei der Ruptur der Tubensehwangerschaft. 263

II. Abschnitt näher an der Mitte der Rupturstelle als in I. Venenruptur und Hämatome (H). Die Vene (V) ist sehr stark erweitert, die Endothelzellen fehlen stellenweise. Die Hämatome sind isoliert.

III. Abschnitt an der Mitte der Rupturstelle. Venenruptur. Hämatome und Platzen nach dem intervillösen Raum. Die Hämatome sind kombiniert.

Figur 4. Mikroskopische Zeichnung der Ränder der Rupturstelle; PeritonealEpithelzellen (P), mittlere Tubenwand (M) und Trophoblast (T).

I. Abschnitt an der Rupturstelle.

II. Abschnitt in der Rupturstelle. 